\title{
Itinerarios didácticos con smartphones para promover la educación ambiental y la competencia digital entre el alumnado de secundaria
}

\author{
Juan-Francisco Álvarez-Herrero \\ juanfran.alvarez@ua.es \\ Universidad de Alicante, España \\ José Hernández-Ortega \\ josehernandezortega@ucm.es \\ Universidad Complutense de Madrid, España
}

\begin{abstract}
Resumen
El alumnado de educación secundaria desconoce en muchas ocasiones la problemática ambiental de su ciudad. Por otro lado, los smartphones gozan de una gran aceptación y uso entre este colectivo. Sin embargo, su uso en las aulas está muy cuestionado. Con la intención de promover una mejora en la educación ambiental y en la competencia digital del alumnado de secundaria en el uso de estos dispositivos se llevó a cabo esta experiencia. Se llevaron a cabo itinerarios didácticos con smartphones por la ciudad de Alcoy en los que se realizaban una serie de pruebas y se hacía uso de la geolocalización, la realidad aumentada, las redes sociales y diversas aplicaciones móviles. Se pasó un cuestionario a los participantes $(\mathrm{N}=447)$ con la idea de conocer el grado de aceptación de la actividad, y se obtuvieron $\mathrm{n}=367$ respuestas. Los resultados demuestran que el alumnado valora notablemente la actividad y sus pruebas. Así mismo, conforme es mayor la edad del alumnado, el interés por la actividad disminuye paulatinamente. Por todo ello, es necesario proponer actividades que busquen la concienciación del alumnado por el medio ambiente, así como el buen uso de los smartphones y sus diversas aplicaciones.
\end{abstract}

\section{Palabras clave}

TIC; tecnología educativa; itinerarios didácticos; educación ambiental; smartphones 


\title{
Didactic itineraries with smartphones to promote environmental education and digital competence among secondary school students
}

\author{
Juan-Francisco Álvarez-Herrero \\ juanfran.alvarez@ua.es \\ Universidad de Alicante, Spain \\ José Hernández-ortega \\ josehernandezortega@ucm.es \\ Universidad Complutense de Madrid, Spain
}

\begin{abstract}
Secondary education students are often unaware of the environmental problems of their city. On the other hand, smartphones are widely accepted and used by this group. However, its use in the classroom is highly questioned. With the intention of promoting an improvement in environmental education and in the digital competence of secondary school students in the use of these devices, this experience was carried out. Didactic itineraries with smartphones were carried out around the city of Alcoy in which a series of tests were carried out and use was made of geolocation, augmented reality, social networks and various mobile applications. A questionnaire was passed to the participants $(N=447)$ with the idea of knowing the degree of acceptance of the activity, and $n=367$ responses were obtained. The results show that the students highly value the activity and its tests. Likewise, as the age of the students is older, the interest in the activity decreases gradually. For all these reasons, it is necessary to propose activities that seek to educate students about the environment, as well as the proper use of smartphones and their various applications.
\end{abstract}

\section{Keywords}

ICT; educational technology; didactic itineraries; environmental education; smartphones 


\section{Introducción}

Nuestra sociedad sigue viviendo de espaldas a una mejor valoración y cuidado del Medio Ambiente. Continuamente estamos ignorando la problemática que supone descuidar este aspecto y que se solucionaría con una mayor atención a la educación ambiental (Severiche-Sierra, GómezBustamante y Jaimes-Morales, 2016). La educación tiene la clave para una mayor implicación y concienciación de la población en esta situación. Urge establecer políticas nacionales o territoriales de educación ambiental que promuevan el desarrollo sostenible en todos los ámbitos de la sociedad (Aguilera, 2018; Annan-Diab y Molinari, 2017; Hopnina, 2020; Sachs et al., 2019).

Sin embargo, el cómo abordar la educación ambiental en los centros educativos sigue siendo a día de hoy una asignatura pendiente (Henderson y Zarger, 2017) y concretamente en España, la escasa presencia de publicaciones sobre la educación ambiental y la educación para la sostenibilidad así lo demuestran (Jiménez-Fontana y García-González, 2017). Nuestra sociedad vive inmersa en un mundo convulso, incierto y ambiguo, donde los avances tecnológicos adquieren un ritmo tan vertiginoso que cuesta seguirlo desde los diferentes ámbitos. La educación no es ajena a estos cambios, pero lejos de dar respuestas, vive sumida en un desconcierto en el que ni las administraciones educativas (sin un criterio de políticas educativas homogéneas y que varían en función de la legislación nacional, autonómica o incluso local) ni muchas veces dentro de un mismo centro educativo (con diferentes enfoques metodológicos y contenidos a trabajan) se sigue una misma dirección. Es por ello, que la educación ambiental, a pesar de su importancia y de la necesidad de ser tratada, aun habiendo muchas buenas prácticas con excelentes resultados (Boca y Saraçlı, 2019), sigue siendo un contenido que muchas veces pasa desapercibido entre el alumnado o no llega a representar un aprendizaje significativo (Valderrama-Hernández, Alcántara y Limón, 2017).

Una solución fácil y cómoda para un correcto tratamiento de la educación ambiental en las aulas, está en la mano del profesorado, y solamente es necesario que se dé cuenta de que la realidad que le es tan cercana, puede y debe ser el motor de este aprendizaje entre su alumnado. El propio contexto o el ambiente más inmediato a una comunidad educativa es el que ofrece un sinfín de oportunidades para ser tratado y estudiado y favorecer una estima y un conocimiento entre el alumnado mucho mayor que otros contenidos que le son ajenos o lejanos (Esteban-Ibáñez, 2017; Lopera, 2017; Dieste, Coma y Blasco-Serrano, 2019; Schild, 2016). Los parques o parajes naturales próximos, las zonas verdes del entorno, la propia localidad, garantizan un profuso conjunto de posibilidades muy ricas en contenidos, matices y en propiciar una valoración y una estima mayor que la que se pudiera derivar en otras opciones no tan cercanas al alumnado (Jose, Patrick y Moseley, 2017). Sin perder de vista que una salida del aula al campo o a la ciudad, garantiza una mayor motivación e implicación del alumnado que conlleva a su vez un aprendizaje verdaderamente significativo, pues este se ha generado desde la ilusión y el interés. Y por otro lado, hablamos de un recurso interdisciplinar y transversal que a su vez sirve como eje vertebrador del currículum de cualquier nivel o etapa educativa (López y Segura, 2013) y que permite alejarnos de la idea de considerar los itinerarios didácticos y las salidas de campo como meros complementos o de ámbito únicamente de la formación informal o extraescolar (Zimmerman, Land y Jung, 2016).

El itinerario didáctico o la salida de campo, se constituye a partir de prácticas educativas que garantizan un aprendizaje activo en el que el alumnado es protagonista en primera persona de cuanto va construyendo, tanto habilidades como competencias que desarrolla de manera significativa (Aguilera, 2018; Alcántara y Medina, 2019). Son recursos que implican cambiar el aula por el mundo exterior para interactuar con una realidad a la que muchas veces, pese a tenerla al alcance de su mano, le es desconocida. 
En edades comprendidas entre los 14 y los 18 años, correspondientes a la etapa educativa de Educación Secundaria Obligatoria, ya se puede apelar a la responsabilidad y autonomía personal del alumnado. Esta generación es la más amplia de la sociedad con acceso a un teléfono móvil con acceso a Internet (Ditrendia, 2019; We are social, 2020). El uso en el aula de dispositivos móviles, ampliamente cuestionado e incluso prohibido en determinados países como Francia (González, 2019), ofrece unas posibilidades muy valiosas para la mejora del aprendizaje. Dichos dispositivos se convierten en excelentes herramientas de aprendizaje, un aprendizaje que posibilita con su conectividad, que pueda ser en cualquier momento y lugar, venciendo así muchas barreras (Gil 2019, Hwang, Zou y Lin, 2020; Lai, 2019; Papadokostaki et al., 2020). Sin embargo, dado que actualmente todavía no hay un uso generalizado de estos en los centros educativos y no se enseña a utilizarlos con intereses pedagógicos, los adolescentes realizan un uso de ellos básicamente centrado en las redes sociales o en el ocio y entretenimiento (Besolí, Palomas y Chamarro, 2018; Salcines-Talledo, González-Fernández y Briones, 2020) desconociendo las amplias posibilidades que para el aprendizaje ofrecen. No es sólo la cantidad de herramientas que engloban (teléfono, grabadora de audio, cámara de fotos y videos, reloj, calculadora, geolocalizador, miniordenador, brújula, atlas, etc.) sino también el amplio abanico de aplicaciones que pueden soportar y que pueden emplearse para aprender y construir conocimiento. Negar o prohibir sus amplias posibilidades en el proceso de enseñanza-aprendizaje supone ir en contra de una realidad que, hoy por hoy, convierte a los smartphones en unos recursos elementales que definen la forma de vida y de comportamiento de las personas, haciendo necesario establecer un nuevo paradigma educativo digital (Coma, 2013; Fernández y Gutiérrez, 2017).

Uniendo de forma combinada diversas variables como son:

- el uso de los dispositivos móviles mediante la utilización de las redes sociales, más concretamente una de las más famosas y utilizadas entre los adolescentes en la actualidad como es Instagram (Pujiati, Zahra y Tamela, 2019; We are social, 2020).

- el uso de aplicaciones y herramientas de dichos dispositivos, como la realidad aumentada, la geolocalización, o el hacer fotos y grabar videos (Ahmad, Ali y Amirudin, 2019; Kalogiannakis y Papadakis, 2017; Maccagno, 2020; Villaluestre y López-Manrique, 2020).

- permitir al alumnado la realización de itinerarios en pequeños grupos con total autonomía por la ciudad donde además van a poder moverse con el transporte urbano (ÁlvarezHerrero, 2016).

- la interacción con el patrimonio, con la cultura y la sociedad local además de la coincidencia de alumnado de su misma edad de otros centros educativos (Bernardo, 2016; Joo, Martínez y García-Bermejo, 2017; Luna, Ibáñez-Etxeberria y Rivero, 2019).

- el desarrollo de habilidades y competencias que van más allá de las que se trabajan en el aula y en una sola asignatura o materia (es decir, hablamos de interdisciplinariedad y de un aprendizaje activo, completo, contextualizado, cercano, real y vivencial) (Díaz, 2020; Torralba y Herrero, 2016).

- incentivar el aprendizaje significativo a través de prácticas transmedia o multimodales en las que la variedad semiótica facilita la adquisición e interacción del alumnado con el conocimiento a través de soportes digitales (Cassany, 2012; Lankshear y Knobel, 2011; Scolari, 2013).

La asociación de profesores Lacenet, lanzó en 2013 el proyecto global World Mobile City Project (WMCP) que consistía en la realización de una serie de yincanas o itinerarios didácticos por la ciudad en la que se daban todas estas variables (Barlam, Ribas, Foixenc y Rochera, 2018; Díaz, 2020). El proyecto adquiría en cada caso un nombre particular atendiendo a la ciudad que cobraba 
el protagonismo, así en Barcelona tiene lugar la Barcelonada, en Manresa la Manresada, o en Alcoy la Alcoyanada. Dichas yincanas se celebran desde entonces ampliando año tras año tanto las sedes en las que se realizan como el número de participantes. Se implica al alumnado de educación secundaria de centros educativos de la ciudad y alrededores y bajo las premisas dadas se celebran en una fecha fijada alrededor de mediados de febrero de cada año. El caso de Alcoy resulta a su vez ser bastante particular, pues cada año o edición, toma como protagonista una temática o ámbito de interés, haciendo girar toda la yincana alrededor de dicho tema o materia. En los últimos años se ha trabajado la poesía y más concretamente la obra del poeta alcoyano Joan Valls (Álvarez-Herrero, 2018), al modernismo, o la historia, arte y cultura alcoyana, por citar algunos ejemplos.

En la edición de 2019, la Alcoyanada optó por hacer protagonista a la educación ambiental de una forma contextualizada, descubriendo aspectos ambientales íntimamente ligados a la ciudad, a su paisaje urbano y natural, a la diversidad natural presente en sus calles, pero también a la contaminación, la sostenibilidad y a los problemas y deficiencias que la ciudad de Alcoy presenta. Se facilitaba de esta manera un aprendizaje móvil, contextualizado, donde además se trabaja de forma cooperativa y fomentando competencias y habilidades como la competencia comunicativa, el aprender a aprender, la socialización, etc. y en el que además se mejoraba el conocimiento y el valor y la estima por el entorno, por la naturaleza presente en su ciudad y una concienciación real sobre los problemas que más directamente afectan a esta.

\section{Objetivos}

Los objetivos de esta investigación pretenden:

1. Conocer el grado de aceptación del alumnado de secundaria a la realización de itinerarios didácticos para promover y motivar su aprendizaje, el desarrollo de su competencia digital y un mejor conocimiento de su entorno.

2. Analizar y comparar el grado de aceptación en la realización de actividades de educación ambiental frente a otras de otra índole en los itinerarios didácticos propuestos.

3. Comprobar si existe una diferencia en el grado de aceptación de este tipo de actividades atendiendo al sexo y la edad del alumnado de secundaria.

\section{Método}

Previo a la realización de la yincana que aquí abordamos, se organizó una formación auspiciada por el Centro de Formación, Innovación y Recursos Educativos (CEFIRE) de Alicante con 30 horas de duración, en la que participaron 30 profesores de 13 centros educativos de educación secundaria de Alcoy y otras localidades próximas como Ibi, Muro de Alcoy, Bañeres de Mariola, Biar, Xixona y Castalla. En dicha formación además de abordar las cuestiones técnicas y de gestión necesarias para llevar a cabo con posterioridad la yincana, se trataron estrategias y dinámicas con las que trabajar y concienciar al alumnado sobre la problemática medioambiental (centrando un mayor interés en: la contaminación acústica, la preservación de la diversidad y más concretamente de la flora urbana, los vertidos incontrolados de residuos sólidos urbanos, etc.) de la ciudad objeto de la yincana. Tanto las cuestiones técnicas y competenciales en tecnología digital como las dinámicas medioambientales fueron llevadas a cabo por el profesorado con su respectivo alumnado a la par 
que se estuvo realizando el curso y previamente a la realización de la yincana. Con ello, se aseguró que el alumnado iba a estar preparado y concienciado para la realización de la actividad.

\section{a. Desarrollo de la actividad}

Cada docente de los participantes en la actividad, en los días previos a la realización de la misma, trabajó con su alumnado los aspectos organizativos y conceptuales necesarios para un buen desempeño de la misma. Al alumnado se les explicó cómo utilizar las diferentes aplicaciones que se iban a emplear, los requisitos que debían cumplir en el uso de sus dispositivos móviles, así como cuestiones relativas al desplazamiento y cómo desenvolverse con autonomía por la ciudad.

En la yincana el alumnado de secundaria, organizado en grupos de 4 a 6 estudiantes, debía moverse por la ciudad de Alcoy bien a pie bien en transporte urbano, con la misión de completar un recorrido con 6 puntos de interés que le eran asignados mediante unos tarjetones ( 6 en total, uno por cada punto de interés) que se les repartía al principio de la jornada y todo ello entre las 9:00 y las 13:00 horas del mismo día. Se cuenta con 60 puntos de interés diferentes, cada uno con su tarjetón diferente, que, al ser mezclados y repartidos aleatoriamente de 6 en 6 a los grupos de alumnado participante, generaban muchas combinaciones y posibilidades de recorridos diferentes. Para desplazarse, cada grupo contaba, además, con una tarjeta de transporte gratuita para ese día con la que podían realizar cuantos viajes necesitasen todas las personas del grupo con los autobuses urbanos. Durante la realización de dicho itinerario, en todo momento se encontraban monitorizados por su profesorado, dado que en sus dispositivos móviles llevaban instalada una aplicación que permitía saber su localización en tiempo real. Cada estudiante participante iba provisto de una chapa identificativa de la actividad y se habían pedido los permisos pertinentes tanto a la Policía Local, como a la Policía Nacional y Guardia Civil de Alcoy. El alumnado, en sus smartphones llevaban también instaladas las aplicaciones necesarias para la realización de una serie de pruebas que debían realizar, una prueba en cada punto de interés, a elegir una de las siguientes en cada punto:

- (V01) Realizar un video en el que a modo de mimo o de pequeño sketch representasen de modo descriptivo, alguna característica del lugar visitado. Por ejemplo, si se trataba del Parque de Bomberos, imitar con gestos y acciones la actuación de los bomberos en la extinción de un incendio.

- (V02) Realizar una video-entrevista a alguna persona que localizasen en el punto de interés visitado y a la que le preguntasen cómo era la escuela de secundaria cuando dicha persona realizó dichos estudios.

- (V03) Realizar un video en el que se pudiese observar cómo se utilizaba la aplicación PlantNet para identificar y catalogar alguna especie vegetal presente en los alrededores del punto de interés.

- (I01) Realizar una imagen en la que detectasen y de esta forma, denunciasen, una barrera arquitectónica presente en las inmediaciones del punto de interés visitado.

- (I02) Realizar una captura de pantalla del smartphone tras hacer uso de la aplicación Sonómetro para comprobar la contaminación acústica presente en el punto de interés.

- (I03) Realizar una imagen de alguna deficiencia en la vía urbana, bien de índole urbanística bien de contaminación vertida en las calles o parques en las proximidades del punto de interés.

Las tres grabaciones de video y las tres imágenes resultantes de su paso por los 6 puntos de interés de su recorrido, debían subirlas desde cuentas en abierto a la red social Instagram y con las 
etiquetas o hashtags de la jornada: \#ALCada19 y \#WMCP19, generando de esta forma un mapa colaborativo y geolocalizado con imágenes y videos de toda la ciudad de Alcoy.

A las 13:00 horas todos los participantes debían reunirse en un pabellón deportivo céntrico de la ciudad, donde una vez presentes todos se procedía a la realización de un pequeño acto de clausura de la jornada con la entrega de un pequeño detalle a los participantes.

Se pidió al profesorado participante que invitase a su alumnado a la cumplimentación de un pequeño cuestionario de evaluación de la actividad en los dos días siguientes a la realización de la yincana. Para ello, el profesorado antes de iniciar con su alumnado la cumplimentación del cuestionario, hizo una pequeña introducción sobre el mismo, y tras la cumplimentación de forma libre y anónima por parte del alumnado, el profesorado realizó también con su alumnado una valoración grupal de la actividad y de si se habían logrado o no los objetivos de la misma desde el punto de vista del alumnado. Las respuestas a dicho cuestionario, así como las dadas en entrevistas que se realizaron a docentes y estudiantes participantes y de las observaciones, impresiones y reflexiones de todos ellos, son las que nos sirven para traer aquí los resultados de nuestra investigación y tratar de dar respuesta a los objetivos que nos hemos marcado.

\section{b. Participantes}

Como ya se ha indicado, previamente a la realización de la yincana se realizó una formación con 30 docentes de 13 centros educativos, y a estos se les unieron 2 docentes más, haciendo que al final el número total de docentes participantes fuese de 32 .

El alumnado participante fue de un total de 447 perteneciente a 13 centros educativos de educación secundaria. 7 de estos centros eran de la propia ciudad de Alcoy (CE01, CE02, CE03, CE04, CE05, CE06 y CE07), y los otros 6 de las ciudades próximas de Bañeres de Mariola, Biar, Castalla, Ibi, Muro de Alcoy y Xixona (CE08, CE09, CE10, CE11, CE12 y CE13).

En el cuestionario de evaluación, del total de estudiantes participantes en la yincana $(N=447)$, dado el carácter voluntario del mismo, participaron $367(n=367)$, un $82.1 \%$ lo que la hace una muestra significativa, con un nivel de confianza del $99 \%$ y un margen de error del $2.87 \%$.

\section{c. Instrumentos de recogida de datos}

Para conocer el grado de aceptación del alumnado sobre este tipo de actividades, se confeccionó ad hoc un cuestionario a través de Google Forms (https://forms.gle/f6cY5fWYdAr98eYi9), donde además de tres preguntas sociodemográficas (sexo, edad y centro educativo al que pertenece), había 7 preguntas más de tipo Likert, graduadas entre el 1 (nada) y el 5 (mucho), y que incidían sobre el grado de aceptación de la actividad en su conjunto y de cada una de las 6 pruebas o actividades realizadas en cada uno de los 6 puntos de interés visitados. Finalmente, el cuestionario incluía tres preguntas abiertas: una de valoración cualitativa global de la actividad por parte del alumnado y otras dos en las que debía destacar aquellos aspectos que más y menos les habían gustado. El cuestionario estuvo disponible en línea en los dos días posteriores a la realización de la yincana y como ya se ha comentado, se obtuvieron 367 respuestas válidas. Los resultados cuantitativos fueron tratados con el programa estadístico IBM SPSS versión 25 y los cualitativos con el programa ATLAS.ti 9.

Por otro lado, se realizaron 5 entrevistas pautadas a 5 docentes de 5 centros participantes y otras 15 entrevistas a 15 estudiantes de 3 de los centros participantes ( 5 estudiantes de cada centro). 


\section{Resultados}

\section{a. Datos sociodemográficos}

De las 367 personas participantes en el cuestionario de evaluación de la actividad, 202 (55\%) fueron mujeres y 165 (45\%) hombres, tal y como se puede ver en la tabla 1.

\begin{tabular}{|c|c|c|}
\hline Sexo & Frecuencia & Porcentaje \\
\hline Hombre & 165 & 45 \\
\hline Mujer & 202 & 55 \\
\hline TOTAL & 367 & 100 \\
\hline
\end{tabular}

Tabla. 1. Sexo de los/las participantes

La edad de los participantes abarca entre los 14 y los 18 años, siendo la edad media de la muestra 15.68 años. Tal y como se observa en la tabla 2, casi la mitad de la muestra (el 49.6\%) son estudiantes de 16 años, siendo la presencia de los de 18 años algo testimonial con tan solo 3 participantes.

\begin{tabular}{|c|c|c|}
\hline Edad & Frecuencia & Porcentaje \\
\hline 14 & 27 & 7.4 \\
\hline 15 & 112 & 30.5 \\
\hline 16 & 182 & 49.6 \\
\hline 17 & 43 & 11.7 \\
\hline 18 & 3 & 0.8 \\
\hline TOTAL & 367 & 100 \\
\hline
\end{tabular}

Tabla. 2. Edad de los/las participantes

Y respecto al alumnado y profesorado participante en la actividad, así como del alumnado participante en el cuestionario de evaluación, en la tabla 3 se puede ver que es bastante variable y dispersa y no sigue ningún criterio lógico.

\begin{tabular}{|c|c|c|c|c|c|c|c|}
\hline $\begin{array}{c}\text { Centro } \\
\text { educativo }\end{array}$ & $\begin{array}{c}\mathrm{N} \\
\text { Alum. }\end{array}$ & $\begin{array}{c}\% \\
\text { Alum. }\end{array}$ & $\begin{array}{c}\mathrm{N} \\
\text { Profes. }\end{array}$ & $\begin{array}{c}\% \\
\text { Profes. }\end{array}$ & $\begin{array}{c}\mathrm{n} \\
\text { Alum. }\end{array}$ & $\begin{array}{c}\% \\
\text { Alum. }\end{array}$ & $\begin{array}{c}\% \\
\text { participación }\end{array}$ \\
\hline CE01 & 14 & 3.1 & 1 & 3.1 & 10 & 2.7 & 71.4 \\
\hline CE02 & 12 & 2.7 & 3 & 9.3 & 6 & 1.6 & 50.0 \\
\hline CE03 & 90 & 20.1 & 4 & 12.5 & 72 & 19.6 & 80.0 \\
\hline CE04 & 35 & 7.8 & 2 & 6.3 & 33 & 9.0 & 94.3 \\
\hline CE05 & 20 & 4.5 & 1 & 3.1 & 14 & 3.8 & 70.0 \\
\hline CE06 & 25 & 5.6 & 1 & 3.1 & 22 & 6.0 & 88.0 \\
\hline CE07 & 11 & 2.4 & 1 & 3.1 & 9 & 2.5 & 81.8 \\
\hline CE08 & 20 & 4.5 & 3 & 9.3 & 14 & 3.8 & 70.0 \\
\hline
\end{tabular}




\begin{tabular}{|c|c|c|c|c|c|c|c|}
\hline CE09 & 18 & 4.0 & 2 & 6.3 & 14 & 3.8 & 77.8 \\
\hline CE10 & 40 & 8.9 & 4 & 12.5 & 28 & 7.6 & 70.0 \\
\hline CE11 & 43 & 9.6 & 4 & 12.5 & 39 & 10.6 & 90.7 \\
\hline CE12 & 24 & 5.4 & 2 & 6.3 & 18 & 4.9 & 75.0 \\
\hline CE13 & 95 & 21.4 & 4 & 12.5 & 88 & 24.0 & 92.6 \\
\hline TOTAL & 447 & 100 & 32 & 100 & 367 & 100 & \\
\hline
\end{tabular}

Tabla. 3. Alumnado y profesorado participante en la actividad y alumnado participante en la evaluación, atendiendo al centro educativo al que pertenecen.

La participación en el cuestionario de evaluación se sitúa en todos los centros educativos por encima del 70\% a excepción de uno de ellos (el CE02) que es del 50\%. Pero como ya hemos comentado, la participación media en este cuestionario es del $82.1 \%$, lo que la hace significativa.

\section{b. Resultados globales}

En la tabla 4 podemos ver los resultados obtenidos a nivel global para el grado de aceptación de la actividad en su conjunto (\#ALCada19) y para cada una de las 6 actividades realizadas en los puntos de interés (V01, V02, V03, I01, I02 y I03). En una primera estimación se observa que la actividad en su conjunto goza de mayor aceptación (4.34) que cuando al alumnado se le pregunta por cada una de las actividades de forma individual (donde todas están por debajo de una puntuación de 4). Y dentro de lo que son las actividades individuales, de entre aquellas en las que se les pedía realizar un video, las actividades V01 y V02 también obtienen mayor puntuación que en las que se les pedía realizar una imagen (I01, I02 y I03) o la del tercer video (V03) que obtiene la puntuación más baja de todas.

\begin{tabular}{|l|c|c|c|c|c|c|c|}
\hline & \#ALCada19 & V01 & V02 & V03 & I01 & I02 & I03 \\
\hline Media & 4.34 & 3.93 & 3.89 & 3.36 & 3.40 & 3.59 & 3.64 \\
\hline $\begin{array}{l}\text { Desviación } \\
\text { típica }\end{array}$ & .671 & 1.098 & 1.072 & 1.201 & 1.195 & 1.163 & 1.148 \\
\hline Varianza & .450 & 1.205 & 1.150 & 1.443 & 1.427 & 1.353 & 1.318 \\
\hline
\end{tabular}

Tabla. 4. Grado de aceptación de la actividad en su conjunto (\#ALCada19) y de cada una de las actividades realizadas de forma individual (V01, V02, V03, I01, I02 y I03).

Si consideramos las actividades I01, I02 y la V03 como aquellas más estrechamente ligadas a la educación ambiental, en una primera estimación, podemos advertir que tienen valores más bajos que las otras tres actividades.

\section{c. Resultados atendiendo a las diferentes variables.}

En la tabla 5 podemos ver qué ocurre con las medias del grado de aceptación de la actividad en su conjunto y para cada una de las actividades, atendiendo al sexo del participante. 


\begin{tabular}{|l|c|c|c|c|c|c|c|}
\hline & \#ALCada19 & V01 & V02 & V03 & I01 & I02 & I03 \\
\hline Hombre & 4.27 & 3.76 & 3.73 & 3.32 & 3.35 & 3.53 & 3.64 \\
\hline Mujer & 4.40 & 4.06 & 4.03 & 3.39 & 3.45 & 3.63 & 3.65 \\
\hline Global & 4.34 & 3.93 & 3.89 & 3.36 & 3.40 & 3.59 & 3.64 \\
\hline
\end{tabular}

Tabla. 5. Grado medio de aceptación de la actividad en su conjunto (\#ALCada19) y de cada una de las actividades realizadas de forma individual (V01, V02, V03, I01, I02 y I03) atendiendo al sexo del participante.

Se puede apreciar que las puntuaciones obtenidas en el caso de las mujeres son siempre más elevadas que las obtenidas en los hombres y que tanto en unas como en otros, se siguen dando menores puntuaciones en las actividades más ligadas a la educación ambiental con respecto a las que tienen menor relación. Igual a como ocurría a nivel global, las dos primeras actividades en las que se pedía realizar un video (V01 y V02), cuentan con mayores puntuaciones tanto en hombres como en mujeres que el resto de actividades.

El análisis de la variable de la edad, nos permite identificar unos resultados heterogéneos como así se puede interpretar en la tabla 6 . Tanto la actividad en su conjunto como todas las actividades a nivel individual, gozan de menor grado de aceptación cuanta mayor es la edad del participante. El alumnado de 14 años obtiene las mejores puntuaciones y éstas descienden de forma inversamente proporcional al incremento de la edad de los participantes. Así mismo, si analizamos cada edad, podemos comprobar que, en todas ellas, a excepción de los de 18 años, se siguen las mismas tendencias que a nivel global. Es decir, las actividades V01 y V02 son siempre las mejor valoradas respecto al resto de actividades, y aquellas que más estrecha relación tienen con la educación ambiental obtienen peores puntuaciones que las otras actividades. El que no se cumpla en la edad de 18 años estas premisas que sí se dan en el resto de edades, encuentra su explicación en la casi testimonial representación que supone los 3 estudiantes que cuentan con esta edad.

\begin{tabular}{|c|c|c|c|c|c|c|c|}
\hline Edad & \#ALCada19 & V01 & V02 & V03 & I01 & I02 & I03 \\
\hline 14 & 4.59 & 4.26 & 4.15 & 3.85 & 3.81 & 3.93 & 4.11 \\
\hline 15 & 4.43 & 3.97 & 3.96 & 3.52 & 3.66 & 3.66 & 3.71 \\
\hline 16 & 4.30 & 3.90 & 3.85 & 3.24 & 3.27 & 3.53 & 3.59 \\
\hline 17 & 4.16 & 3.77 & 3.81 & 3.14 & 3.09 & 3.44 & 3.47 \\
\hline 18 & 4.00 & 3.33 & 2.67 & 3.00 & 2.67 & 3.00 & 3.00 \\
\hline Global & 4.34 & 3.93 & 3.89 & 3.36 & 3.40 & 3.59 & 3.64 \\
\hline
\end{tabular}

Tabla. 6. Grado medio de aceptación de la actividad en su conjunto (\#ALCada19) y de cada una de las actividades realizadas de forma individual (V01, V02, V03, I01, I02 y I03) atendiendo a la edad del participante.

\section{d. Resultados cualitativos de las preguntas abiertas}

En las preguntas abiertas que recogían tanto los aspectos positivos como a mejorar que el alumnado destacaba de la actividad y la valoración global de la misma, obtenemos información muy interesante sobre la percepción del alumnado tanto en si consideran esta actividad como una práctica que mejora su aprendizaje, como si ayuda en el desarrollo de su competencia digital y en 
su concienciación sobre la problemática medioambiental de su entorno más inmediato.

Se han codificado y categorizado las respuestas con carácter positivo dadas y los resultados obtenidos tras contabilizarlas los podemos apreciar en la tabla 7.

\begin{tabular}{|l|c|c|}
\hline & Frecuencia & Porcentaje \\
\hline Genera interés y motivación por aprender & 323 & 88.0 \\
\hline Mejora el aprendizaje & 117 & 31.9 \\
\hline Concienciación medioambiental & 281 & 76.6 \\
\hline Desarrollo de la competencia digital & 297 & 80.9 \\
\hline Desarrollo de habilidades sociales & 184 & 50.1 \\
\hline Carácter lúdico & 312 & 85.0 \\
\hline
\end{tabular}

Tabla. 7. Aspectos valorados positivamente por el alumnado

Así mismo, se realizó con aquellas cuestiones que el alumnado consideró que debían mejorarse de esta actividad, y los resultados podemos observarlos en la tabla 8.

\begin{tabular}{|l|c|c|}
\hline & Frecuencia & Porcentaje \\
\hline Nada & 246 & 67.0 \\
\hline Esfuerzo físico (tener que caminar) & 27 & 7.4 \\
\hline $\begin{array}{l}\text { Los premios (no estar de acuerdo con los premios } \\
\text { concedidos, falta de premios para todos los participantes) }\end{array}$ & 16 & 367 \\
\hline $\begin{array}{l}\text { Duración de la actividad (muy corta, que sólo se haga una } \\
\text { vez en el curso académico, etc.) }\end{array}$ & 41 & 11.2 \\
\hline Las pruebas (aburridas, poco interesantes) & 6 & 1.6 \\
\hline $\begin{array}{l}\text { La organización de la actividad (explicaciones aportadas, } \\
\text { gestión, horarios, etc.) }\end{array}$ & 4 & 1.1 \\
\hline
\end{tabular}

Tabla. 8. Aspectos valorados negativamente por el alumnado

Tanto de los resultados de las respuestas positivas como de las negativas (donde 246 estudiantes respondieron que no encontraban nada negativo en la actividad), podemos afirmar que la actividad es valorada muy positivamente por el alumnado, quien destaca que esta actividad genera interés y motivación por aprender ya que, entre otras cuestiones, favorece una mayor conciencia medioambiental, un desarrollo de la competencia digital y el desarrollo de habilidades sociales.

\section{e. Resultados de las entrevistas}

Las entrevistas realizadas tanto a docentes (5) como a estudiantes participantes en la yincana (15) vienen a confirmar los resultados obtenidos en las respuestas al cuestionario.

El profesorado confirma que trabajó tanto con anterioridad como con posterioridad a la actividad, conceptos y dinámicas para poder sacar un mayor provecho de la actividad, tanto en el desarrollo de la competencia digital como de la concienciación medioambiental que de esta se desprende.

$\mathrm{Y}$ el alumnado, confirma con sus respuestas en las entrevistas, que vieron esta actividad como muy 
interesante y facilitadora de una mayor motivación hacia el aprendizaje de cuestiones relacionadas con su entorno más próximo, con una mejor socialización y sentimiento de ciudadanía.

\section{Discusión}

La utilización de itinerarios didácticos mediante el uso de dispositivos móviles y recursos de realidad aumentada, geolocalización y redes sociales, es una buena práctica educativa que conduce a percepciones bastante positivas entre el alumnado de educación secundaria. En este tipo de actividades no solo se consigue un desarrollo competencial en una realidad más cercana al alumnado, sino que además origina una mejora en la motivación y el interés por el aprendizaje. Así se desprende de los resultados que recogemos en esta investigación, en la que el alumnado de secundaria valora muy positivamente este tipo de actividades y que están de acuerdo con otros estudios similares realizados bien con alumnado de secundaria o bien con alumnado universitario (Alcántara y Medina, 2019; Cheng, Hwang y Chen, 2019; Martín, 2016; Montero y Jerez, 2019; San Pedro et al., 2018), y que constatan que este tipo de proyectos permite el desarrollo de múltiples competencias así como su carácter interdisciplinar posibilita el acercamiento y aprendizaje de diversas disciplinas. Sólo un pequeño porcentaje, un $12 \%$, no encuentra la actividad motivadora o con el suficiente interés por aprender.

Una valoración notable que es ligeramente mayor en el caso de las mujeres respecto a los hombres y que pierde valor a mayor edad entre la franja comprendida entre los 14 y los 18 años, edad del alumnado que aquí nos ocupa. Pese a que no se da plenamente, sí puede detectarse que el alumnado da prioridad al uso por afinidad y motivación a las actividades mediadas por el vídeo frente a las centradas el en único uso de la imagen y el texto.

Hemos observado que la valoración de las actividades realizadas en estos itinerarios didácticos ligadas a la educación ambiental y contextualizadas en la realidad de la ciudad en la que tienen lugar, es positiva para todos los casos y en todas las variables. Nuevamente se obtienen mejores puntuaciones entre las mujeres que entre los hombres, y estas también son mayores a menor edad del alumnado. Sin embargo, al compararlas con otras actividades ya no tan estrechamente ligadas a la educación ambiental, se observa que las primeras obtienen peores valoraciones respecto a estas últimas. Encontramos una explicación para ello en que las actividades no tan estrechamente ligadas a la educación ambiental en esta yincana son por un lado más lúdicas y atractivas al alumnado, pues no conllevan un complemento académico (realizar un mimo o sketch, realizar una video-entrevista a una persona de la ciudad, etc.); y por otro lado, las actividades ligadas a la educación ambiental requieren en dos de ellas el manejo de una aplicación en sus dispositivos, y también en dos de ellas se les pide una imagen, frente a los dos videos que se les piden en las otras, cuando ya hemos comentado que el alumnado de secundaria valora más positivamente la utilización del video frente a la utilización de la imagen fija. Perspectiva que corrobora la tendencia del alumnado por el uso de actividades mediadas por el vídeo ante otros formatos estáticos como el texto o la imagen fija (Cassany, 2012; Sáez-Bondía y Cortes-Gracia, 2019; Valverde-Crespo et al., 2018).

Tras ver y analizar los resultados obtenidos se nos plantea una cuestión acerca de la idoneidad o no de la tecnología digital, y más concretamente del uso de los dispositivos móviles y aplicaciones y recursos de realidad aumentada, geolocalización y redes sociales, a la hora de establecer puentes o nexos de unión entre el aprendizaje del alumnado y su aprecio y respeto por el medio ambiente. Al igual que destacan Buchanan, Pressick-Kilborn y Maher (2019), pese a tener limitaciones y no obtener resultados excelentes, entendemos que el uso de las tecnologías digitales ayuda a motivar, 
a valorar y a tomar conciencia del medio ambiente y a ver en la sostenibilidad una salida a los problemas que este plantea. Asimismo, Ayerbe y Perales (2020) también comprobaron con éxito cómo el trabajo a través del Aprendizaje Basado en Proyectos (ABP) en el medio ambiente urbano y con alumnado de secundaria producía una mejora en los niveles de conciencia del alumnado.

\section{Conclusiones y futuras líneas de investigación.}

Llevar el aprendizaje a la calle, al contexto y la realidad más cercana al alumnado, siempre ha sido un recurso con el que se han experimentado resultados positivos. Hacerlo además acompañado con un uso apropiado y coherente de las tecnologías digitales, reporta mayores beneficios cuando además estamos hablando de alumnado de educación secundaria y del uso de los smartphones y de las redes sociales. Y combinar todo ello junto con la concienciación y la valoración del medio ambiente, haciendo ver la problemática con la que nos encontramos y promoviendo la sostenibilidad como solución posible, permite llevar adelante una actividad completa en la que, si bien las valoraciones del alumnado no son excelentes, sí nos permiten allanar el camino hacia la formación de personas competentes, ciudadanos del mundo.

La introducción de mejoras en la preparación del profesorado participante en cuestiones ambientales a tratar en la actividad, así como en la propuesta didáctica realizada a través de actividades basadas en el desarrollo de competencias y estrategias participativas lúdicas, permitiría la obtención de unos mejores resultados. Por todo ello, desde aquí proponemos para futuras actividades, la adecuación de estas y otras variables que permitan de esta actividad unos mejores resultados orientados a una mayor concienciación y valoración de las cuestiones medioambientales más próximas y de mayor interés para el alumnado de secundaria.

Tal y como ha quedado demostrado, la realización de este tipo de actividades es valorada muy positivamente por parte del alumnado. Se requiere de una mayor implicación, que pasaría por una mayor interacción y carga sentimental y emotiva. Cambiar el estatismo del aula para llevar la ciencia a la calle, consigue que el alumnado la vea como tangible, algo en lo que le llama implicarse y que permite que el aprendizaje se produzca de forma significativa puesto que se realiza desde hechos vivenciales que ha experimentado el alumnado en primera persona. Sin embargo, como ya hemos mencionado, no podemos caer en la banalidad de tratar estas actividades como meras salidas del aula, sin contenido ni sentido, pues entonces sí estaríamos convirtiendo una oportunidad de aprendizaje en un acto lúdico y contraproducente para el desarrollo y construcción de aprendizaje.

Entre las futuras líneas de investigación que nos proponemos llevar a cabo están: rediseñar la actividad en su conjunto para que disponga de actividades competenciales ligadas a la educación ambiental y las tecnologías digitales con mayor carga de interacción y de motivación en el alumnado; conocer los motivos por los que el profesorado no realiza, o si las realiza, por qué no lo hace con mayor frecuencia, este tipo de prácticas educativas con su alumnado; obtener muestras más amplias involucrando a todas las ciudades que realizan yincanas dentro del proyecto global del WMCP y que permitan observar si se producen diferencias notables entre los resultados obtenidos en los diferentes contextos en los que se han llevado a cabo; analizar los discursos visuales del alumnado para establecer patrones semióticos en su práctica digital; y propiciar con este tipo de actividades que la implicación del alumnado con la sostenibilidad del medio ambiente vaya más allá de esta actividad y haga que requiera un compromiso o servicio por su parte para con la comunidad. 


\section{Referencias}

Aguilera, D. (2018). La salida de campo como recurso didáctico para enseñar ciencias. Una revisión sistemática. Revista Eureka sobre Enseñanza y Divulgación de las Ciencias, 15(3), 3103. http://dx.doi.org/ $10.25267 /$ Rev_Eureka_ensen_divulg_cienc.2018.v15.i3.3103

Aguilera Peña, R. (2018). La educación ambiental, una estrategia adecuada para el desarrollo sostenible de las comunidades. Revista DELOS Desarrollo Local Sostenible, 31. Recuperado de: http://hdl.handle.net/ 20.500.11763/delos31roberto-aguilera

Ahmad, A. F. A, Ali, K. N., y Amirudin, R. (2019). Evaluating students readiness, expectancy, acceptance and effectiveness of augmented reality based construction technology education. International Journal of Built Environment and Sustainability, 6(1), 7-13. https://doi.org/10.11113/ijbes.v6.n1.309

Alcántara, J. y Medina, S. (2019). El uso de los itinerarios didácticos (SIG) en la educación ambiental. Enseñanza de las Ciencias, 37(2), 173-188. https://doi.org/10.5565/rev/ensciencias.2258

Álvarez-Herrero J. F. (2016). Aprendizaje móvil con geolocalización y Realidad Aumentada en la realización de itinerarios didácticos. En R. Roig-Vila (Ed.), EDUcación y TECnología. Propuestas desde la investigación y la innovación educativa, (pp. 13-14). Barcelona: Octaedro.

Álvarez-Herrero J. F. (2018). La poesía y la competencia digital de los alumnos de secundaria. En E. Álvarez y J. Blasco (eds.), Humanidades Digitales. Retos, Recursos y Nuevas Propuestas, (pp. 121-126). Valladolid: Agilice Digital.

Annan-Diab, F., y Molinari, C. (2017). Interdisciplinarity: Practical approach to advancing education for sustainability and for the Sustainable Development Goals. The International Journal of Management Education, 15(2B), 73-83. https://doi.org/10.1016/j.ijme.2017.03.006

Ayerbe, J. y Perales, F. J. (2020). Reinventa tu ciudad: aprendizaje basado en proyectos para la mejora de la conciencia ambiental en estudiantes de Secundaria. Enseñanza de las Ciencias, 38(2), 181-203. https://doi.org/10.5565/rev/ensciencias.2812

Barlam, R., Ribas, M., Foixenc, N. y Rochera, M. J. (2018). Dispositivos móviles y personalización del aprendizaje. La Manresada, mi Manresada. En C. Coll (coord.), Dosier Graó no 3: La personalización del aprendizaje. Barcelona: Graó.

Bernardo, C. (2016). Itinerario didáctico en Monte Naranco: el respeto al medio ambiente como nexo de unión intercultural (Trabajo Final de Máster). Universidad de Oviedo, Oviedo.

Besolí, G., Palomas, N. y Chamarro, A. (2018). Uso del móvil en padres, niños y adolescentes: Creencias acerca de sus riesgos y beneficios. Aloma: Revista de Psicologia, Ciències de l'Educació i de l'Esport, 36(1), 29-39. Recuperado de: http://revistaaloma.net/index.php/aloma/article/view/328

Boca, G. D. y Saraçlı, S. (2019). Environmental Education and Student's Perception, for Sustainability. Sustainability, 11, 1553. https://doi.org/10.3390/su11061553

Buchanan, J., Pressick-Kilborn, K. y Maher, D. (2019). Promoting Environmental Education for Primary Schoolaged Students Using Digital Technologies. Eurasia Journal of Mathematics, Science and Technology Education, 15(2), em1661. https://doi.org/10.29333/ejmste/100639

Cassany, D. (2012). En_línea: leer y escribir en la red. Anagrama.

Cheng, S. C., Hwang, G. J. y Chen, C. H. (2019). From reflective observation to active learning: A mobile experiential learning approach for environmental science education. British Journal of Educational Technology, 50(5), 2251-2270. https://doi.org/10.1111/bjet.12845

Coma, L. (2013). Dinamizar y digitalizar la ciudad: itinerarios urbanos, dispositivos móviles y códigos QR. Her\&Mus. Heritage \& Museography, 13, 63-68. Recuperado de: https://www.raco.cat/index.php/ Hermus/article/view/313395

Díaz, S. M. (2020). Pedagogías emergentes y exploración del contexto con realidad aumentada: un estudio de caso múltiple (Tesis doctoral). Universidad de Murcia, Murcia. Recuperado de: http://hdl.handle.net/ 


\section{$10201 / 87013$}

Dieste, B., Coma, T. y Blasco-Serrano, A. C. (2019). Inclusión de los objetivos de desarrollo sostenible en el currículum de educación primaria y secundaria en escuelas rurales de Zaragoza. Revista Internacional de Educación para la Justicia Social, 8(1), 97-115. https://doi.org/10.15366/riejs2019.8.1.006

Ditrendia (2019). Informe Ditrendia: Mobile en España y en el Mundo. Madrid: Ditrendia Digital Marketing Trends. Recuperado de: https://tinyurl.com/tvhstc4

Esteban-Ibáñez, M. (2017). Educación Ambiental. Evolución y desarrollo en el contexto reglado escolar español. Runae, Monográfico no 1, 221-240. Recuperado de: http://revistas.unae.edu.ec/index.php/runae/ article/view/172

Fernández Rodríguez, E. y Gutiérrez Pequeño, J. M. (2017). La socialización de los jóvenes interconectados: Experimentando la identidad en la sociedad aumentada. Profesorado. Revista de Currículum y Formación de Profesorado, 21(2), 171-190. Recuperado de: https://recyt.fecyt.es/index.php/ profesorado/article/view/59455

Gil, J. (2019). Interconectados apostando por la construcción colectiva del conocimiento. Aprendizaje móvil en Educación Infantil y Primaria. Píxel-Bit. Revista de Medios y Educación, 54, 185-203. https://doi.org/ 10.12795/pixelbit.2019.i54.10

González, F. (2019). La prohibición del teléfono móvil en las escuelas en Francia. Revista de Administración Pública, 208, 379-401. https://doi.org/10.18042/cepc/rap.208.13Yyyy

Henderson, J. A. y Zarger, R. K. (2017). Toward political ecologies of environmental education. The Journal of Environmental Education, 48(4), 285-289. http://doi.org/10.1080/00958964.2017.1336978

Hopnina, H. (2020). Education for the future? Critical evaluation of education for sustainable development goals. The Journal of Environmnetal Education, 51(4), 280-291. https://doi.org/ 10.1080/00958964.2019.1710444

Hwang, G. J., Zou, D. y Lin, J. (2020). Effects of a multi-level concept mapping-based question-posing approach on students' ubiquitous learning performance and perceptions. Computers \& Education, 149, 103815. https://doi.org/10.1016/j.compedu.2020.103815

Jiménez-Fontana, R. y García-González, E. (2017). Visibilidad de la Educación Ambiental y la Educación para la sostenibilidad en las publicaciones españolas sobre educación científica. Revista Eureka sobre Enseñanza y Divulgación de las Ciencias, 14(1), 271-285. http://dx.doi.org/10.25267/ Rev_Eureka_ensen_divulg_cienc.2017.v14.i1.20

Joo, J., Martínez, F. y García-Bermejo, J. R. (2017). Realidad Aumentada y Navegación Peatonal Móvil con contenidos Patrimoniales: Percepción del aprendizaje. RIED. Revista Iberoamericana de Educación a Distancia, 20(2), 93-118. http://dx.doi.org/10.5944/ried.20.2.17602

Jose, S., Patrick, P. G. y Moseley, C. (2017). Experiential learning theory: the importance of outdoor classrooms in environmental education. International Journal of Science Education, Part B: Communication and Public Engagement, 7(3), 269-284. https://doi.org/10.1080/21548455.2016.1272144

Kalogiannakis, M. y Papadakis, S. (2017). Combining mobile technologies in environmental education: a Greek case study. International Journal of Mobile Learning and Organization, 11(2), 108-130.

Lai, C. L. (2019). Trends of mobile learning: A review of the top 100 highly cited papers. British Journal of Educational Technology, O(0), 1-22. https://doi.org/10.1111/bjet.12884

Lankshear, C., y Knobel, M. (2011). New literacies. McGraw-Hill Education (UK).

Lopera, M. (2017). Experiencia de formación del profesorado basada en el contexto ciudad-escuela. Revista Iberoamericana de Educación, 74(1), 41-58. Recuperado de: https://rieoei.org/historico/documentos/ 7730.pdf

López, F. y Segura, J. A. (2013). Los itinerarios didácticos: un recurso interdisciplinar y vertebrador del curriculum. Espiral. Cuadernos del profesorado, 6(12), 15-31. http://dx.doi.org/10.25115/ 


\section{ecp.v6i12.954}

Luna, U., Ibáñez-Etxeberria, A., y Rivero, M. P. (2019). El patrimonio aumentado. 8 apps de Realidad Aumentada para la enseñanza-aprendizaje del patrimonio. Revista interuniversitaria de formación del profesorado, 33(1), 43-62. https://doi.org/10.47553/rifop.v33i1.72088

Maccagno, A. P. (2020). Itinerarios interactivos con realidad aumentada en procesos de evaluación. Reflexión Académica en Diseño y Comunicación, 43, 228-235.

Martín, S. (2016). Propuesta metodológica para el diseño de itinerarios didácticos de Ciencias de la Naturaleza. Didácticas Específicas, 14, 144-150. Recuperado de: http://hdl.handle.net/10486/671862

Montero, J. y Jerez, O. (2019). The teaching itinerary as a resource for development of communication competences: design of a bilingual app. En J. V. Salido y P. V. Salido (Coords.), La competencia lingüística en la comunicación: visiones multidisciplinares y transversalidad. Cuenca: Ediciones de la Universidad de Castilla-La Mancha. http://doi.org/10.18239/jor_19.2019.02

Papadokostaki, K., Panagiotakis, S., Malamos, A. y Vassilakis, K. (2020). Mobile Learning in the Era of IoT: Is Ubiquitous Learning the Future of Learning? En S. Papadakis \& M. Kalogiannakis (Eds.), Mobile Learning Applications in Early Childhood Education. Hershey, PA, USA: IGI Global. http://doi.org/ 10.4018/978-1-7998-1486-3.ch013

Pujiati, H., Zahra y Tamela, E. (2019). The Use of Instagram to Increase Students' Motivation and Students' Competence in Learning English. En 1st International Conference on Education Social Sciences and Humanities (ICESSHum 2019). https://doi.org/10.2991/icesshum-19.2019.103

Sachs, J. D., Schmidt-Traub, G., Mazzucato, M., Messner, D., Nakicenovic, N., y Rockström, J. (2019). Six Transformations to achieve the Sustainable Development Goals. Nature Sustainability, 2, 805-814. https://doi.org/10.1038/s41893-019-0352-9

Sáez-Bondía, M. J., y Cortés-Gracia, A. L. (2019). ¿Cómo cambian las ideas de los estudiantes de Máster de Profesorado sobre una actividad práctica de campo tras su vídeo-análisis y discusión en pequeños grupos? Revista Eureka sobre Enseñanza y Divulgación de las Ciencias, 16(2), 2602. http://doi.org/ 10.25267/Rev_Eureka_ensen_divulg_cienc.2019.v16.i2.2602

Salcines-Talledo, I., González-Fernández, N. y Briones, E. (2020). The Smartphone as a Pedagogic Tool. Student Profiles as related to its Use and Knowledge. Journal of New Approaches in Educational Research, 9(1), 91-109. http://dx.doi.org/10.7821/naer.2020.1.454

San Pedro, M. B., López, I., Fombella, I., del Cura, Y., Sánchez, B. y Álvarez, A. I. (2018). Social Sciences, Art and Physical Activity in Leisure Environments. An Inter-Disciplinary Project for Teacher Training. Sustainability, 10(6), 1786. http://doi.org/10.3390/su10061786

Schild, R. (2016). Environmental citizenship: What can political theory contribute to environmental education practice? The Journal of Environmental Education, 47(1), 19-34. http://doi.org/ $10.1080 / 00958964.2015 .1092417$

Scolari, C. (2013). Narrativas transmedia. Cuando todos los medios cuentan. Deusto.

Severiche-Sierra, C., Gómez-Bustamante, E. y Jaimes-Morales, J. (2016). La educación ambiental como base cultural y estrategia para el desarrollo sostenible. Telos: Revista de Estudios Interdisciplinarios en Ciencias Sociales, 18(2), 266-281. Recuperado de: http://ojs.urbe.edu/index.php/telos/article/view/ $\underline{753}$

Torralba, A. y Herrero, M. (2016). El aprendizaje móvil (mlearning) aplicado a la Didáctica del Medio Natural en la formación inicial de Maestros en Educación Primaria y en Educación Infantil. EDUNOVATIC2016, I Congreso Virtual Internacional sobre Educación, Innovación y TIC, 502-507. Recuperado de: http:// hdl.handle.net/10651/39323

Valderrama-Hernández, R., Alcántara, L. y Limón, D. (2017). The complexity of environmental education: teaching ideas and strategies from teachers. Procedia-Social and Behavioral Sciences, 237, 968-974. https://doi.org/10.1016/j.sbspro.2017.02.137 
Valverde-Crespo, D., de Pro-Bueno, A., y González-Sánchez, J. (2018). La competencia informacional-digital en la enseñanza y aprendizaje de las ciencias en la educación secundaria obligatoria actual: una revisión teórica. Revista Eureka sobre Enseñanza y Divulgación de las Ciencias, 15(2), 2105. http://doi.org/ 10.25267/Rev Eureka_ensen_divulg_cienc.2018.v15.i2.2105

Villalustre, L. y López-Manrique, I. (2020). Geolocation for the Improvement of Spatial and Naturalist Intelligence in Primary Education. En Examining Multiple Intelligences and Digital Technologies for Enhanced Learning Opportunities. Hershey, PA, USA: IGI Global. http://doi.org/ 10.4018/978-1-7998-0249-5.ch008

We are social (enero, 2020). Digital 2020 España [Mensaje en un blog]. Recuperado de: https://tinyurl.com/ rgok6kd

Zimmerman, H. T., Land, S. M. y Jung, Y. J. (2016). Using Augmented Reality to Support Children's Situational Interest and Science Learning During Context-Sensitive Informal Mobile Learning. En A. Peña-Ayala (Eds.), Mobile, Ubiquitous, and Pervasive Learning. Advances in Intelligent Systems and Computing, vol 406. Cham, Suiza: Springer. https://doi.org/10.1007/978-3-319-26518-6_4 